ALGEBRA, LOGIC AND NUMBER THEORY

BANACH CENTER PUBLICATIONS, VOLUME 121

INSTITUTE OF MATHEMATICS

POLISH ACADEMY OF SCIENCES

WARSZAWA 2020

\title{
EXTENSIONS OF A VALUATION FROM $K$ TO $K[x]$
}

\author{
JOSNEI NOVACOSKI \\ Departamento de Matemática-UFSCar \\ Rodovia Washington Luís, 235, 13565-905 São Carlos, SP \\ ORCID:0000-0002-6810-0994Ｅ-mail: josnei@dm.ufscar.br
}

\begin{abstract}
In this paper we give an introduction on how one can extend a valuation from a field $K$ to the polynomial ring $K[x]$ in one variable over $K$. This follows a similar line as the one presented by the author in his talk at ALaNT 5 . We will discuss the objects that have been introduced to describe such extensions. We will focus on key polynomials, pseudo-convergent sequences and minimal pairs. Key polynomials have been introduced and used by various authors in different ways. We discuss these works and the relation between them. We also discuss a recent version of key polynomials developed by Spivakovsky. This version provides some advantages that will be discussed in this paper. For instance, it allows us to relate key polynomials, in an explicit way, to pseudo-convergent sequences and minimal pairs. This paper also provides examples that illustrate these objects and their properties. Our main goal when studying key polynomials is to obtain more accurate results on the problem of local uniformization. This problem, which is still open in positive characteristic, was the main topic of the paper of the author and Spivakovsky in the proceedings of ALaNT 3.
\end{abstract}

1. Introduction. If $\nu_{0}$ is a valuation on a field $K$, then what are the possible extensions $\nu$ of $\nu_{0}$ to $K[x]$ ? This question has been extensively studied and many objects have been introduced to describe such extensions. Three of the more relevant are key polynomials, pseudo-convergent sequences and minimal pairs. The main goal of this paper is to describe these objects and present the relation between them.

2010 Mathematics Subject Classification: 13A18.

Key words and phrases: valuations, key polynomials, pseudo-convergent sequences, minimal pairs. During part of the realization of this project the author was supported by a research grant from Fundação de Amparo à Pesquisa do Estado de São Paulo (process number 2017/17835-9). The paper is in final form and no version of it will be published elsewhere. 
Throughout this paper, we will fix the following notation and assumptions:

$$
\begin{cases}\frac{K}{K} & \text { is a field, } \\ K[x] & \text { is a fixed algebraic closure of } K, \\ \nu & \text { is a valuation on } K[x] \\ \mu & \text { is an extension of } \nu \text { to } \bar{K}[x] .\end{cases}
$$

We start by defining key polynomials. These objects were introduced by MacLane in [5] and refined by Vaquié in [9. The definition that we present here is slightly different and is due to Spivakovsky. The basic properties of Spivakovsky's key polynomials were developed in [7] and will be summarized in Section 3. In Section 2 we will discuss the MacLane-Vaquié key polynomials and in Section 3 we discuss how they are related to Spivakovsky's key polynomials.

For a positive integer $b$ and $f \in K[x]$ let $\partial_{b} f$ be the $b$-th formal derivative of $f$, i.e., $\partial_{b} f$ are the uniquely determined polynomials for which the Taylor expansion

$$
f(x)-f(a)=\sum_{i=1}^{\operatorname{deg}(f)} \partial_{i} f(a)(x-a)^{i},
$$

is satisfied for every $a \in K$. An easy and useful formula for computing $\partial_{b} f$ is

$$
\partial_{b} f=\frac{1}{b !} \frac{\partial^{b}}{\partial x^{b}}(f)
$$

(Observe that the expression above makes sense even in positive characteristic because $b$ ! divides the integer obtained by performing $b$ many times the derivative of $f$ with respect to $x$.) For a polynomial $f \in K[x]$ let

$$
\epsilon(f)=\max _{b \in \mathbb{N}}\left\{\frac{\nu(f)-\nu\left(\partial_{b} f\right)}{b}\right\} .
$$

A monic polynomial $Q \in K[x]$ is said to be a (Spivakovsky's) key polynomial for $\nu$ if for every $f \in K[x]$,

$$
\epsilon(f) \geq \epsilon(Q) \Longrightarrow \operatorname{deg}(f) \geq \operatorname{deg}(Q) .
$$

In [3], Kaplansky introduced the concept of pseudo-convergent sequences. For a valued field $(K, \nu)$, a pseudo-convergent sequence is a well-ordered subset $\left\{a_{\rho}\right\}_{\rho<\lambda}$ of $K$, without last element, such that

$$
\nu\left(a_{\sigma}-a_{\rho}\right)<\nu\left(a_{\tau}-a_{\sigma}\right) \text { for all } \rho<\sigma<\tau<\lambda .
$$

Let $R$ be a ring with $K \subseteq R$ and consider an extension of $\nu$ to $R$, which we call again $\nu$. An element $a \in R$ is said to be a limit of $\left\{a_{\rho}\right\}_{\rho<\lambda} \subseteq K$ if for every $\rho<\lambda$ we have $\nu\left(a-a_{\rho}\right)=\nu\left(a_{\rho+1}-a_{\rho}\right)$.

One of the main goals of [7] is to compare key polynomials and pseudo-convergent sequences. These results are presented in Section 5 .

Another theory that has been developed to study extensions of a given valuation to the ring of polynomials in one variable is the theory of minimal pairs of definition of a valuation (see [1]). A minimal pair for $\nu$ is a pair $(a, \delta) \in \bar{K} \times \mu(\bar{K}[x])$ such that for every $b \in \bar{K}$

$$
\mu(b-a) \geq \delta \Longrightarrow[K(b): K] \geq[K(a): K]
$$


If in addition,

$$
\mu(x-a)=\delta \geq \mu(x-b)
$$

for every $b \in \bar{K}$, then $(a, \delta)$ is called a minimal pair of definition for $\nu$.

The main goal of [6] is to compare key polynomials and minimal pairs. These relations will be presented in Section 5 .

For a valued field $(K, \nu)$ we denote by $K \nu$ the residue field and by $\nu K$ the value group of $\nu$, respectively. A valuation $\nu$ on $K[x]$ is called valuation-algebraic if $\nu(K(x)) / \nu K$ is a torsion group and $K(x) \nu \mid K \nu$ is an algebraic extension. Otherwise, it is called valuation-transcendental. If $\nu$ is valuation-transcendental, then it is residue-transcendental if $K(x) \nu \mid K \nu$ is a transcendental extension and value-transcendental if $\nu(K(x)) / \nu K$ is not a torsion group.

Given two polynomials $f, q \in K[x]$ with $q$ monic, we call the $q$-expansion of $f$ the expression

$$
f(x)=f_{0}(x)+f_{1}(x) q(x)+\ldots+f_{n}(x) q^{n}(x)
$$

where for each $i, 0 \leq i \leq n, f_{i}=0$ or $\operatorname{deg}\left(f_{i}\right)<\operatorname{deg}(q)$. For a polynomial $q(x) \in K[x]$, the $q$-truncation of $\nu$ is defined as

$$
\nu_{q}(f):=\min _{0 \leq i \leq n}\left\{\nu\left(f_{i} q^{i}\right)\right\}
$$

where $f=f_{0}+f_{1} q+\ldots+f_{n} q^{n}$ is the $q$-expansion of $f$.

We point out that the original definition of minimal pairs, presented in [1, is slightly different than the one appearing here. The reason is that, with the original definition, one can prove that a valuation on $K[x]$ admits a pair of definition if and only if it is residue-transcendental. On the other hand, from the results in [7, one can prove that an extension admits a minimal pair of definition (as presented here) if and only if it is valuation-transcendental. Hence, with our definition we are considering all the valuations which are somehow simpler to handle (i.e., valuations for which the sequence of key polynomials has a last element). This result will follow from the following:

THEOREM 1.1 (Theorem 1.3 of [6]). A valuation $\nu$ on $K[x]$ is valuation-transcendental if and only if there exists a polynomial $q \in K[x]$ such that $\nu=\nu_{q}$.

The theorem above can be seen as the version of Theorem 3.11 of [4] for key polynomials and truncations. In Section 3 , we describe a complete sequence of key polynomials for $\nu$. If $\boldsymbol{Q}$ is such a sequence and $Q$ is a largest element for it, then $\nu=\nu_{Q}$. Hence, we conclude from Theorem 1.1 that if $\boldsymbol{Q}$ has a last element, then $\nu$ is valuation-transcendental.

This paper is divided as follows. In Section 2, we describe the theory of MacLaneVaquié key polynomials. In Section 3, we describe some of the most important properties of Spivakovsky's key polynomials. Also in Section 3, we describe the relation of MacLaneVaquié and Spivakovsky's key polynomials. In Section 4 we describe some of the main properties of pseudo-convergent sequences. Section 5 is devoted to presenting the comparison between these three objects. In Section 6, we present an example that illustrates the theory.

Acknowledgements. I thank the anonymous referee for a careful reading and for providing useful suggestions in the notation and presentation of this paper. 
2. Key polynomials. Take a commutative $\operatorname{ring} R$ and an ordered abelian group $\Gamma$. Take $\infty$ to be an element not in $\Gamma$ and set $\Gamma_{\infty}$ to be $\Gamma \cup\{\infty\}$ with extensions of addition and order as usual (i.e., $\infty>\gamma$ for every $\gamma \in \Gamma$ and $\infty+\gamma=\infty$ for every $\gamma \in \Gamma$ ).

Definition 2.1. A valuation on $R$ is a map $\nu: R \longrightarrow \Gamma_{\infty}$ such that:

(V1) $\nu(a b)=\nu(a)+\nu(b)$ for every $a, b \in R$,

(V2) $\nu(a+b) \geq \min \{\nu(a), \nu(b)\}$ for every $a, b \in R$,

(V3) $\nu(1)=0$ and $\nu(0)=\infty$.

One can show that under the assumptions (V1) and (V2), the condition (V3) is equivalent to the support of $\nu$, defined by $\operatorname{supp}(\nu):=\{a \in R \mid \nu(a)=\infty\}$, being a prime ideal of $R$. Hence, if $R$ is a field, then (V3) is equivalent to

$$
\nu(x)=\infty \Longleftrightarrow x=0,
$$

which is the usual assumption for valuations defined on a field.

REMARK 2.2. We use this opportunity to correct a mistake in the definition of a valuation in [6]. There we require that $\operatorname{supp}(\nu)$ is a minimal prime ideal, when it should be a prime ideal.

REMARK 2.3. If $R=K[x]$, then valuations on $R$ describe all the valuations extending $\nu_{0}=\left.\nu\right|_{K}$ to simple extensions $K(a)$ of $K$. Indeed, if $\operatorname{supp}(\nu)$ is the zero ideal, then $\nu$ extends in an obvious way to $K(x)$ where $x$ is a transcendental element. If

$$
\operatorname{supp}(\nu) \neq(0) \text {, }
$$

then there exists $p(x) \in K[x]$ monic and irreducible such that $\operatorname{supp}(\nu)=(p)$. Hence, $\nu$ defines a valuation on

$$
K[x] /(p)=K(a)
$$

for some element $a \in \bar{K}$ with minimal polynomial $p(x)$.

Let $\nu_{0}$ be a valuation of $K$ and $\nu$ a valuation of $K[x]$ extending $\nu_{0}$. If $\gamma_{0}=\nu(x)$, then we define

$$
\nu_{1}\left(a_{0}+a_{1} x+\ldots+a_{r} x^{r}\right)=\min \left\{\nu_{0}\left(a_{i}\right)+i \gamma_{0}\right\} .
$$

If $\nu=\nu_{1}$, then we are done. If not, then take a polynomial $\phi_{1}$ of smallest degree such that

$$
\gamma_{1}:=\nu\left(\phi_{1}\right)>\nu_{1}\left(\phi_{1}\right) \text {. }
$$

For each $f \in K[x]$, write $f=f_{0}+f_{1} \phi_{1}+\ldots+f_{r} \phi_{1}^{r}$, with $\operatorname{deg}\left(f_{i}\right)<\operatorname{deg}\left(\phi_{1}\right)$ and define

$$
\nu_{2}(f)=\min \left\{\nu_{1}\left(f_{i}\right)+i \gamma_{1}\right\} .
$$

If $\nu=\nu_{2}$, then we are done. Otherwise we continue the process.

QUESTION 2.4. Can we construct a "sequence" of polynomials $\phi_{i}$ such that $\nu$ is the "limit" of the maps $\nu_{i}$ ?

Key polynomials were first introduced by MacLane in [5]. In order to define MacLane key polynomials, we will need to define the graded algebra associated to a valuation. Let $R$ be a ring and $\nu$ a valuation on $R$. For every $\beta \in \nu R$, set

$$
P_{\beta}:=\{y \in R \mid \nu(y) \geq \beta\} \text { and } P_{\beta}^{+}:=\{y \in R \mid \nu(y)>\beta\} .
$$


The graded algebra of $\nu$ is defined as

$$
\operatorname{gr}_{\nu}(R):=\bigoplus_{\beta \in \nu R} P_{\beta} / P_{\beta}^{+}
$$

For an element $y \in R$ we denote by $\operatorname{in}_{\nu}(y)$ the image of $y \operatorname{in} \operatorname{gr}_{\nu}(R)$, i.e.,

$$
\operatorname{in}_{\nu}(y):=y+P_{\nu(y)}^{+} \in P_{\nu(y)} / P_{\nu(y)}^{+} \subset \operatorname{gr}_{\nu}(R) .
$$

Let $K$ be a field and let $\nu$ be a valuation on $K[x]$, the polynomial ring in one variable over $K$. Given $f, g \in K[x]$, we say that $f$ is $\nu$-equivalent to $g$ (and write $f \sim_{\nu} g$ ) if $\operatorname{in}_{\nu}(f)=\operatorname{in}_{\nu}(g)$. Moreover, we say that $g \nu$-divides $f$ (and write $\left.g\right|_{\nu} f$ ) if there exists $h \in K[x]$ such that $f \sim_{\nu} g \cdot h$.

Definition 2.5. A monic polynomial $\phi \in K[x]$ is a MacLane-Vaquié key polynomial for $\nu$ if it is $\nu$-irreducible (i.e., $\left.\left.\phi\right|_{\nu} f \cdot g \Longrightarrow \phi\right|_{\nu} f$ or $\left.\phi\right|_{\nu} g$ ) and if for every $f \in K[x]$

$$
\left.\phi\right|_{\nu} f \Longrightarrow \operatorname{deg}(f) \geq \operatorname{deg}(\phi) .
$$

Let $\phi$ be a key polynomial for $\nu, \Gamma^{\prime}$ be a group extension of $\nu(K[x])$ and $\gamma \in \Gamma^{\prime}$ such that $\gamma>\nu(\phi)$. For every $f \in K[x]$, let

$$
f=f_{0}+f_{1} \phi+\ldots+f_{n} \phi^{n}
$$

be the $\phi$-expansion of $f$. Define the map

$$
\nu^{\prime}(f):=\min _{0 \leq i \leq n}\left\{\nu\left(f_{i}\right)+i \gamma\right\} .
$$

TheOREM 2.6 (Theorem 4.2 of [5]). The map $\nu^{\prime}$ is a valuation on $K[x]$.

Definition 2.7. The map $\nu^{\prime}$ is called an augmented valuation and denoted by

$$
\nu^{\prime}:=\left[\nu ; \nu^{\prime}(\phi)=\gamma\right] .
$$

Given a valuation $\nu$ on $K$, a group $\Gamma^{\prime}$ containing $\nu K$ and $\gamma \in \Gamma^{\prime}$ we define the map

$$
\nu_{\gamma}\left(a_{0}+a_{1} x+\ldots+a_{n} x^{n}\right):=\min _{0 \leq i \leq n}\left\{\nu\left(a_{i}\right)+i \gamma\right\} .
$$

THEOREM 2.8 (Theorem 4.1 of [5]). The map $\nu_{\gamma}$ is a valuation on $K[x]$.

This valuation is called a monomial valuation and denoted by

$$
\nu^{\prime}:=\left[\nu ; \nu^{\prime}(x)=\gamma\right] .
$$

Consider now the set $\mathcal{V}$ of all valuations on $K[x]$ (extending a fixed valuation $\nu_{0}$ on $K)$. The theorems above give us an algorithm to build valuations on $K[x]$. Namely, take a group $\Gamma_{1}$ containing $\nu(K)$ and $\gamma_{1} \in \Gamma_{1}$. Set

$$
\nu_{1}:=\left[\nu_{0} ; \nu_{1}(x)=\gamma_{1}\right] .
$$

Now, let $\phi_{1}$ be a key polynomial for $\nu_{1}, \Gamma_{2}$ an extension of $\Gamma_{1}$ and $\gamma_{2} \in \Gamma_{2}$ with $\gamma_{2}>\nu_{1}\left(\phi_{1}\right)$. Set

$$
\nu_{2}:=\left[\nu_{1} ; \nu_{2}\left(\phi_{1}\right)=\gamma_{2}\right]
$$


Proceeding iteratively, we build

$$
\begin{array}{ll}
\text { groups } & \nu(K) \subseteq \Gamma_{1} \subseteq \Gamma_{2} \subseteq \ldots \subseteq \Gamma_{n} \subseteq \ldots, \\
\text { valuations } & \nu_{1}, \nu_{2}, \ldots, \nu_{n}, \ldots \in \mathcal{V}, \\
\text { polynomials } \phi_{1}, \ldots, \phi_{n}, \ldots \in K[x]
\end{array}
$$

and $\gamma_{i} \in \Gamma_{i}, i \in \mathbb{N}$, such that $\phi_{i+1}$ is a key polynomial for $\nu_{i}$ and

$$
\nu_{i+1}=\left[\nu_{i} ; \nu_{i+1}\left(\phi_{i}\right)=\gamma_{i+1}\right] .
$$

Assume that we have constructed an infinite sequence as above. If for every $f \in K[x]$ there exists $n_{f} \in \mathbb{N}$ such that $\nu_{n}(f)=\nu_{n_{f}}(f)$ for every $n \geq n_{f}$, then we define

$$
\nu_{\infty}(f):=\nu_{n_{f}}(f) .
$$

On the other hand, if $\Gamma_{n} \subseteq \mathbb{R}$ for every $n \in \mathbb{N}$, then for every polynomial $f \in K[x]$ the sequence $s:=\left\{\nu_{n}(f)\right\}_{n \in \mathbb{N}}$ has a supremum, and since $s$ is increasing we have

$$
\nu_{\infty}(f):=\sup \left\{\nu_{n}(f)\right\}=\lim _{n \rightarrow \infty} \nu_{n}(f) .
$$

Observe that $\nu_{\infty}(f)$ can be $\infty$, even if $f \neq 0$.

TheOREM 2.9 (Theorem 6.2 of [5]). The map $\nu_{\infty}$ is a valuation of $K[x]$.

The valuation in the theorem above is called a limit valuation (and we write $\nu_{\infty}=$ $\left.\lim \nu_{i}\right)$.

Consider now the subset $\mathcal{V}^{c}$ of $\mathcal{V}$ consisting of monomial, augmented and limit valuations (extending $\nu_{0}$ ).

QUESTION 2.10. Is it true that $\mathcal{V}^{c}=\mathcal{V}$ ? In other words, given any valuation $\nu \in \mathcal{V}$, does there exist a sequence of valuations $\nu_{1}, \nu_{2}, \ldots, \nu_{n}, \ldots$ such that $\nu=\nu_{i}$ for some $i$ or $\nu=\lim \nu_{i}$ ?

Let $\nu$ be any valuation on $K[x]$. We put $\nu_{0}:=\left.\nu\right|_{K}$ and

$$
\nu_{1}=\left[\nu_{0} ; \nu_{1}(x)=\nu(x)\right] .
$$

If $\nu=\nu_{1}$, then $\nu \in \mathcal{V}^{c}$ (because it is monomial). If not, then take $\phi_{1} \in K[x]$, monic and of smallest degree among polynomials $f$ satisfying $\nu_{1}(f)<\nu(f)$. One can prove that $\phi_{1}$ is a key polynomial for $\nu_{1}$. Consider then the valuation

$$
\nu_{2}=\left[\nu_{1} ; \nu_{2}\left(\phi_{1}\right)=\nu\left(\phi_{1}\right)\right] .
$$

If $\nu_{2}=\nu$, then $\nu \in \mathcal{V}^{c}$ (because is an augmented valuation). If not, then we choose $\phi_{2} \in K[x]$ monic and of smallest degree among polynomials satisfying $\nu_{2}(f)<\nu(f)$. Again, one can prove that $\phi_{2}$ is a key polynomial for $\nu_{2}$ and consider

$$
\nu_{3}=\left[\nu_{2} ; \nu_{3}\left(\phi_{2}\right)=\nu\left(\phi_{2}\right)\right] .
$$

We proceed iteratively until we find a valuation $\nu_{n}$ with $\nu_{n}=\nu$, or constructing an infinite sequence $\left\{\nu_{i}\right\}_{i \in \mathbb{N}}$ such that $\nu_{i} \neq \nu$ and $\nu_{i+1}$ is an augmented valuation of $\nu_{i}$. We have the following:

THEOREM 2.11 (Theorem 8.1 of [5]). If $\nu_{0}$ is a discrete valuation of $K$ of rank one, and the infinite sequence above has been constructed, then $\nu=\lim \nu_{i}$. In particular, if every valuation of $K$ is discrete, then $\mathcal{V}^{c}=\mathcal{V}$. 
REMARK 2.12. Observe that if $\nu_{0}$ is discrete of rank one, then $\Gamma_{n} \subseteq \mathbb{R}$ and we can always construct $\lim _{n \rightarrow \infty} \nu_{n}$.

If $\nu_{0}$ is not discrete, then $\mathcal{V}^{c}$ does not have to be equal to $\mathcal{V}$ (as will be shown in Section 6. This happens because we might need a sequence of key polynomials of order type greater than $\omega$. In order to find a sequence of "augmented" valuations for a given valuation, Vaquié introduced "limit key valuations" (associated to limit key polynomials).

Vaquié's idea was to continue the algorithm started above. If the produced sequence of valuations $\left\{\nu_{n}\right\}$ is not enough to define the valuation (i.e., if $\nu$ is not the "limit" of the sequence $\left\{\nu_{n}\right\}$ ), then we pick a polynomial of smallest degree for which the sequence $\left\{\nu_{n}(f)\right\}$ is bounded and not ultimately constant, and start the process over again (such a polynomial will be a limit key polynomial). Since the degree of this new polynomial must be greater than the degree of the key polynomials appearing before, this process will eventually stop. In the next paragraphs, we present the formal definitions of family of augmented iterated valuations, which extend the notion of the sequence $\left\{\nu_{n}\right\}$ presented above.

More precisely, a family $\left\{\nu_{\alpha}\right\}_{\alpha \in A}$ of valuations of $K[x]$, indexed by a totally ordered set $A$, is called a family of augmented iterated valuations if for all $\alpha$ in $A$, except $\alpha$ the smallest element of $A$, there exists $\theta$ in $A, \theta<\alpha$, such that the valuation $\nu_{\alpha}$ is an augmented valuation of the form $\nu_{\alpha}=\left[\nu_{\theta} ; \nu_{\alpha}\left(\phi_{\alpha}\right)=\gamma_{\alpha}\right]$, and if we have the following properties:

- If $\alpha$ admits an immediate predecessor in $A$, then $\theta$ is that predecessor, and in the case when $\theta$ is not the smallest element of $A$, the polynomials $\phi_{\alpha}$ and $\phi_{\theta}$ are not $\nu_{\theta}$-equivalent and satisfy $\operatorname{deg}\left(\phi_{\theta}\right) \leq \operatorname{deg}\left(\phi_{\alpha}\right)$;

- if $\alpha$ does not have an immediate predecessor in $A$, then for all $\beta$ in $A$ such that $\theta<\beta<\alpha$, the valuations $\nu_{\beta}$ and $\nu_{\alpha}$ are equal to the augmented valuations

$$
\nu_{\beta}=\left[\nu_{\theta} ; \nu_{\beta}\left(\phi_{\beta}\right)=\gamma_{\beta}\right] \text { and } \nu_{\alpha}=\left[\nu_{\beta} ; \nu_{\alpha}\left(\phi_{\alpha}\right)=\gamma_{\alpha}\right],
$$

respectively, and the polynomials $\phi_{\alpha}$ and $\phi_{\beta}$ have the same degree.

For $f, g \in K[x]$, we say that $f A$-divides $g\left(\left.f\right|_{A} g\right)$ if there exists $\alpha_{0} \in A$ such that $\left.f\right|_{\nu_{\alpha}} g$ for every $\alpha \in A$ with $\alpha>\alpha_{0}$. A polynomial $\phi$ is said to be $A$-minimal if for any polynomial $f \in K[x]$ if $\left.\phi\right|_{A} f$, then $\operatorname{deg}(\phi) \leq \operatorname{deg}(f)$. Also, we say that $\phi$ is $A$-irreducible if for every $f, g \in K[x]$, if $\left.\phi\right|_{A} f \cdot g$, then $\left.\phi\right|_{A} f$ or $\left.\phi\right|_{A} g$.

Definition 2.13. A monic polynomial $\phi$ of $K[x]$ is said to be a MacLane-Vaquié limit key polynomial for the family $\left\{\nu_{\alpha}\right\}_{\alpha \in A}$ if it is $A$-minimal and $A$-irreducible.

Let $\left\{\nu_{\alpha}\right\}_{\alpha \in A}$ be a family of iterated valuations of $K[x]$ and, for each $\alpha \in A$, denote the value group of $\nu_{\alpha}$ by $\Gamma_{\nu_{\alpha}}$. Then

$$
\Gamma_{A}:=\bigcup_{\alpha \in A} \Gamma_{\nu_{\alpha}}
$$

is a totally ordered abelian group. For a polynomial $f \in K[x]$, the family $\left\{\nu_{\alpha}\right\}_{\alpha \in A}$ is said to be convergent for $f$ if $\left\{\nu_{\alpha}(f)\right\}$ is unbounded in $\Gamma_{A}$ or there exists $\alpha_{f} \in A$ such that $\nu_{\alpha}(f)=\nu_{\alpha_{f}}(f)$ for every $\alpha \geq \alpha_{f}$. If $\left\{\nu_{\alpha}\right\}_{\alpha \in A}$ is convergent for every $f \in K[x]$, then we define

$$
\lim _{\alpha \in A} \nu_{\alpha}(f):=\sup _{\alpha \in A} \nu_{\alpha}(f)
$$


Observe that $\lim _{\alpha \in A} \nu_{\alpha}(f)=\infty$ if $\left\{\nu_{\alpha}(f)\right\}$ is unbounded and is equal to $\nu_{\alpha_{f}}(f)$, otherwise.

THEOREM 2.14 (Théorème 2.4 of [9]). Let $\nu$ be a valuation of $K[x]$ extending a valuation $\nu_{0}$ of $K$. Then, there exists a family of iterated valuations $\left\{\nu_{\alpha}\right\}_{\alpha \in A}$ of $K[x]$, convergent for every $f \in K[x]$, such that

$$
\nu(f)=\lim _{\alpha \in A} \nu_{\alpha}(f) .
$$

REMARK 2.15. Theorem 2.14 is a generalization of Theorem 2.11 The difference is that, if $\nu$ is not discrete, then we might need a sequence of key polynomials with order type greater than $\omega$.

3. Spivakovsky's key polynomials. We start this section by presenting a characterization of $\epsilon(f)$ in terms of the fixed extension $\mu$ of $\nu$ to $\bar{K}[x]$. For a monic polynomial $f \in K[x]$, we define

$$
\delta(f):=\max \{\mu(x-a) \mid a \text { is a root of } f\} .
$$

EXAMPLE 3.1. Let $f(x)=\left(x-a_{1}\right)\left(x-a_{2}\right)\left(x-a_{3}\right)$. Then

$$
\begin{aligned}
& \partial_{1}(f)=\left(x-a_{1}\right)\left(x-a_{2}\right)+\left(x-a_{1}\right)\left(x-a_{3}\right)+\left(x-a_{2}\right)\left(x-a_{3}\right) \\
& \partial_{2}(f)=\left(x-a_{1}\right)+\left(x-a_{2}\right)+\left(x-a_{3}\right) \\
& \partial_{3}(f)=1 .
\end{aligned}
$$

(i) Assume that $\mu\left(x-a_{i}\right)=i$, for $i=1,2,3$, then

$$
\nu(f)=6, \quad \nu\left(\partial_{1} f\right)=3, \quad \nu\left(\partial_{2} f\right)=1 \text { and } \nu\left(\partial_{3} f\right)=0,
$$

and hence

$$
\begin{aligned}
\epsilon(f) & =\max \left\{\frac{\nu(f)-\nu\left(\partial_{1} f\right)}{1}, \frac{\nu(f)-\nu\left(\partial_{2} f\right)}{2}, \frac{\nu(f)-\nu\left(\partial_{3} f\right)}{3}\right\} \\
& =\max \left\{3, \frac{5}{2}, 2\right\}=3=\delta(f) .
\end{aligned}
$$

(ii) Assume that $\mu\left(x-a_{1}\right)=1$ and $\mu\left(x-a_{2}\right)=\mu\left(x-a_{3}\right)=2$, then

$$
\nu(f)=5, \quad \nu\left(\partial_{1} f\right) \geq 3, \quad \nu\left(\partial_{2} f\right)=1 \text { and } \nu\left(\partial_{3} f\right)=0,
$$

and hence

$$
\begin{aligned}
\epsilon(f) & =\max \left\{\frac{\nu(f)-\nu\left(\partial_{1} f\right)}{1}, \frac{\nu(f)-\nu\left(\partial_{2} f\right)}{2}, \frac{\nu(f)-\nu\left(\partial_{3} f\right)}{3}\right\} \\
& =\max \left\{2,2, \frac{5}{3}\right\}=2=\delta(f) .
\end{aligned}
$$

The examples above can be generalized to prove the following.

Proposition 3.2 (Proposition 3.1 of [6]). Let $f \in K[x]$ be a monic polynomial. Then $\delta(f)=\epsilon(f)$.

In particular, $\delta(f)$ does not depend on the choice of the extension $\mu$ of $\nu$ to $\bar{K}[x]$.

Let $q \in K[x]$ be any polynomial. Then $\nu_{q}$ does not need to be a valuation (Example 2.5 of [7]). The first important property of key polynomials is the following. 
Proposition 3.3 (Proposition 2.6 of [7]). If $Q$ is a key polynomial, then $\nu_{Q}$ is a valuation.

We observe that the converse of the above proposition is not true, i.e., there exists a valuation $\nu$ on $K[x]$ and polynomial $q \in K[x]$ such that $\nu_{q}$ is a valuation, but $q$ is not a key polynomial (Corollary 2.4 of [6]).

For a key polynomial $Q \in K[x]$, let

$$
\begin{gathered}
\alpha(Q):=\min \left\{\operatorname{deg}(f) \mid \nu_{Q}(f)<\nu(f)\right\}, \text { and } \\
\Psi(Q):=\left\{f \in K[x] \mid f \text { is monic, } \nu_{Q}(f)<\nu(f) \text { and } \alpha(Q)=\operatorname{deg}(f)\right\} .
\end{gathered}
$$

THEOREM 3.4 (Theorem 2.12 of [7]). A monic polynomial $Q$ is a key polynomial if and only if there exists a key polynomial $Q_{-} \in K[x]$ such that either $Q \in \Psi\left(Q_{-}\right)$or the following conditions are satisfied:

(K1) $\alpha\left(Q_{-}\right)=\operatorname{deg}\left(Q_{-}\right)$,

(K2) the set $\left\{\nu\left(Q^{\prime}\right) \mid Q^{\prime} \in \Psi\left(Q_{-}\right)\right\}$does not contain a maximal element,

(K3) $\nu_{Q^{\prime}}(Q)<\nu(Q)$ for every $Q^{\prime} \in \Psi\left(Q_{-}\right)$,

(K4) $Q$ has the smallest degree among polynomials satisfying (K3).

Definition 3.5. A key polynomial $Q$ is called a (Spivakovsky's) limit key polynomial if the conditions (K1)-(K4) of the theorem above are satisfied.

For a set $\boldsymbol{Q} \subseteq K[x]$ we denote by $\mathbb{N}^{\boldsymbol{Q}}$ the set of mappings $\lambda: \boldsymbol{Q} \longrightarrow \mathbb{N}$ such that $\lambda(q)=0$ for all but finitely many $q \in \boldsymbol{Q}$. For $\lambda \in \mathbb{N}^{\boldsymbol{Q}}$ we denote

$$
\boldsymbol{Q}^{\lambda}:=\prod_{q \in \boldsymbol{Q}} q^{\lambda(q)} \in K[x] .
$$

Definition 3.6. A set $\boldsymbol{Q} \subseteq K[x]$ is called a complete set for $\nu$ if for every $p \in K[x]$ there exists $q \in Q$ such that

$$
\operatorname{deg}(q) \leq \operatorname{deg}(p) \text { and } \nu(p)=\nu_{q}(p) .
$$

Proposition 3.7. If $\boldsymbol{Q} \subseteq K[x]$ is a complete set for $\nu$, then for every $p \in K[x]$ there exist $a_{1}, \ldots, a_{r} \in K$ and $\lambda_{1}, \ldots, \lambda_{r} \in \mathbb{N}^{Q}$, such that

$$
p=\sum_{i=1}^{r} a_{i} \boldsymbol{Q}^{\lambda_{i}} \text { with } \nu\left(a_{i} \boldsymbol{Q}^{\lambda_{i}}\right) \geq \nu(p) \text {, for every } i, 1 \leq i \leq r,
$$

and the elements $Q \in \boldsymbol{Q}$ appearing in the decomposition of $p$ (i.e., for which $\lambda_{i}(Q) \neq 0$ for some $i, 1 \leq i \leq r)$ have degree smaller than or equal to $\operatorname{deg}(p)$. In particular, for every $\beta \in \nu(K[x])$, the additive group $P_{\beta}$ is generated by the elements $a Q^{\lambda} \in P_{\beta}$ where $a \in K$ and $\lambda \in \mathbb{N}^{Q}$.

REMARK 3.8. The latter condition in the proposition above appears in [8] as the definition of generating sequence. The proof of Proposition 3.7 and the Proposition 3.9 below will appear in a forthcoming paper.

The next result gives us a converse for Proposition 3.7

Proposition 3.9. Assume that $\boldsymbol{Q}$ is a subset of $K[x]$ with the following properties:

- $\nu_{Q}$ is a valuation for every $Q \in Q$; 
- for every finite subset $\mathcal{F} \subseteq \boldsymbol{Q}$, there exists $Q \in \mathcal{F}$ such that $\nu_{Q}\left(Q^{\prime}\right)=\nu\left(Q^{\prime}\right)$ for every $Q^{\prime} \in \mathcal{F}$

- for every $p \in K[x]$ there exist $a_{1}, \ldots, a_{r} \in K$ and $\lambda_{1}, \ldots, \lambda_{r} \in \mathbb{N}^{\boldsymbol{Q}}$ such that

$$
p=\sum_{i=1}^{r} a_{i} \boldsymbol{Q}^{\lambda_{i}} \text { with } \nu\left(a_{i} \boldsymbol{Q}^{\lambda_{i}}\right) \geq \nu(p) \text {, for every } i, 1 \leq i \leq r,
$$

and $\operatorname{deg}(Q) \leq \operatorname{deg}(p)$ for every $Q \in \boldsymbol{Q}$ for which $\lambda_{i}(Q) \neq 0$ for some $i, 1 \leq i \leq r$.

Then $\boldsymbol{Q}$ is a complete set for $\nu$.

THEOREM 3.10 (Theorem 1.1 of [7]). Let $\nu$ be a valuation on $K[x]$. Then there exists a set $\boldsymbol{Q} \subseteq K[x]$ of key polynomials, well-ordered (with the order $Q<Q^{\prime}$ if and only if $\left.\epsilon(Q)<\epsilon\left(Q^{\prime}\right)\right)$, such that $\boldsymbol{Q}$ is a complete set for $\nu$.

REMARK 3.11. The definition of complete set of key polynomials presented in [7] does not require that the degree of the polynomial $Q$ for which $\nu_{Q}(p)=\nu(p)$ is smaller than or equal to $\operatorname{deg}(p)$. This assumption is important and we use this opportunity to fix the definition presented there. The proof presented in [7] guarantees that this additional property is satisfied, hence the theorem above is still valid.

The relation between the key polynomials of Spivakovsky and those of MacLaneVaquié is given by the following.

Theorem 3.12 (Theorem 23 of [2]). Let $Q$ be a Spivakovsky's key polynomial for $\nu$. Then $Q$ is a MacLane-Vaquié key polynomial for $\nu_{Q}$.

We also have the following.

TheOrem 3.13 (Theorem 26 of [2]). Let $Q$ and $Q^{\prime}$ be two Spivakovsky's key polynomials for $\nu$ such that $Q^{\prime} \in \Psi(Q)$. Then $Q^{\prime}$ is a MacLane-Vaquié key polynomial for $\nu_{Q}$.

As for the converse, we have:

Theorem 3.14 (Corollary 29 of [2]). Let $Q$ be a MacLane-Vaquié key polynomial for $\nu$ and $\nu^{\prime}$ a valuation of $K[x]$ for which $\nu^{\prime}(Q)>\nu(Q)$ and $\nu^{\prime}(f)=\nu(f)$ for every $f \in K[x]$ with $\operatorname{deg}(f)<\operatorname{deg}(Q)$. Then $Q$ is a Spivakovsky's key polynomial for $\nu^{\prime}$.

From now on, by key polynomial we will mean Spivakovsky's key polynomial, unless stated explicitly.

4. Pseudo-convergent sequences. Let $\left\{a_{\rho}\right\}_{\rho<\lambda}$ be a pseudo-convergent sequence for $(K, \nu)$. For every polynomial $f(x) \in K[x]$, there exists $\rho_{f}<\lambda$ such that either

$$
\nu\left(f\left(a_{\sigma}\right)\right)=\nu\left(f\left(a_{\rho_{f}}\right)\right) \text { for every } \rho_{f} \leq \sigma<\lambda,
$$

or

$$
\nu\left(f\left(a_{\sigma}\right)\right)>\nu\left(f\left(a_{\rho}\right)\right) \text { for every } \rho_{f} \leq \rho<\sigma<\lambda .
$$

Definition 4.1. A pseudo-convergent sequence $\left\{a_{\rho}\right\}_{\rho<\lambda}$ is said to be of transcendental type if for every polynomial $f(x) \in K[x]$ condition $(3)$ holds. Otherwise, $\left\{a_{\rho}\right\}_{\rho<\lambda}$ is said to be of algebraic type. 
The next two theorems justify the definitions of algebraic and transcendental pseudoconvergent sequences.

THEOREM 4.2 (Theorem 2 of [3]). If $\left\{a_{\rho}\right\}_{\rho<\lambda}$ is a pseudo-convergent sequence of transcendental type, without a limit in $K$, then there exists an immediate transcendental extension $K(z)$ of $K$ defined by setting $\nu(f(z))$ to be the value $\nu\left(f\left(a_{\rho_{f}}\right)\right)$ as in condition (3). Moreover, for every valuation $\mu$ in some extension $K(u)$ of $K$, if $u$ is a pseudo-limit of $\left\{a_{\rho}\right\}_{\rho<\lambda}$, then there exists a value preserving $K$-isomorphism from $K(u)$ to $K(z)$ taking $u$ to $z$.

THEOREM 4.3 (Theorem 3 of [3] ). Let $\left\{a_{\rho}\right\}_{\rho<\lambda}$ be a pseudo-convergent sequence of algebraic type, without a limit in $K, q(x)$ a polynomial of smallest degree for which (4) holds and $z$ a root of $q(x)$. Then there exists an immediate algebraic extension of $K$ to $K(z)$ defined as follows: for every polynomial $f(x) \in K[x]$, with $\operatorname{deg} f<\operatorname{deg} q$ we set $\nu(f(z))$ to be the value $\nu\left(f\left(a_{\rho_{f}}\right)\right.$ ) as in condition (3). Moreover, if $u$ is a root of $q(x)$ and $\mu$ is some extension $K(u)$ of $K$ making $u$ a pseudo-limit of $\left\{a_{\rho}\right\}_{\rho<\lambda}$, then there exists a value preserving $K$-isomorphism from $K(u)$ to $K(z)$ taking $u$ to $z$.

5. Comparison results. In this section we describe explicitly the relation between key polynomials, pseudo-convergent sequences and minimal pairs.

THEOREM 5.1 (Theorem 1.2 of [7]). Let $\left\{a_{\rho}\right\}_{\rho<\lambda} \subset K$ be a pseudo-convergent sequence, without a limit in $K$, for which $x$ is a limit. If $\left\{a_{\rho}\right\}_{\rho<\lambda}$ is of transcendental type, then

$$
\boldsymbol{Q}:=\left\{x-a_{\rho} \mid \rho<\lambda\right\}
$$

is a complete set of key polynomials for $\nu$. On the other hand, if $\left\{a_{\rho}\right\}_{\rho<\lambda}$ is of algebraic type, then every polynomial $q(x)$ of minimal degree among the polynomials not fixed by $\left\{a_{\rho}\right\}_{\rho<\lambda}$ is a limit key polynomial for $\nu$.

The theorem above gives us a way to interpret pseudo-convergent sequences in terms of key polynomials. The next theorem gives us a way to obtain the opposite relation.

Proposition 5.2 (Proposition 1.2 of [6]). Let $\boldsymbol{Q}$ be a complete sequence of key polynomials for $\nu$, without last element. For each $Q \in \boldsymbol{Q}$, let $a_{Q} \in \bar{K}$ be a root of $Q$ such that $\mu\left(x-a_{Q}\right)=\delta(Q)$. Then $\left\{a_{Q}\right\}_{Q \in Q}$ is a pseudo-convergent sequence of transcendental type, without a limit in $\bar{K}$, such that $x$ is a limit for it.

We also want to describe the relation between key polynomials and minimal pairs. The next result gives us such a relation.

THEOREM 5.3 (Theorem 1.1 of [6]). Let $Q \in K[x]$ be a monic irreducible polynomial and choose a root a of $Q$ such that $\mu(x-a)=\delta(Q)$. Then $Q$ is a key polynomial for $\nu$ if and only if $(a, \delta(Q))$ is a minimal pair for $\nu$. Moreover, $(a, \delta(Q))$ is a minimal pair of definition for $\nu$ if and only if $\nu=\nu_{Q}$.

6. Example. The main goal of this section is to present an example that will illustrate the objects introduced in the previous sections (i.e., pseudo-convergent sequences, key polynomials, truncations, etc.). The process used in its construction is a matter of research at the moment, and we hope to make it general in the near future. 
Let $k$ be an algebraically closed field of characteristic $p>0$ (e.g., $k=\overline{\mathbb{F}_{p}}$ ) and $K=k(y)^{1 / p^{\infty}}$ the perfect hull of $k(y)$ inside of $k\left(\left(y^{\mathbb{Q}}\right)\right)$. Set $\nu_{0}$ to be the valuation on $K$ induced by the $y$-adic valuation $\nu_{y}$ on $k\left(\left(y^{\mathbb{Q}}\right)\right)$.

6.1. Construction of the first limit key polynomial. Let $x$ be an indeterminate over $K$ and extend $\nu_{0}$ to $K[x]$ by setting

$$
\nu_{1}\left(a_{0}+a_{1} x+\ldots+a_{n} x^{n}\right):=\min _{0 \leq i \leq n}\left\{\nu_{0}\left(a_{i}\right)-\frac{i}{p}\right\} .
$$

In the MacLane-Vaquié's language, we see that $\nu_{1}$ is the monomial valuation given by

$$
\nu_{1}=\left[\nu_{0} ; \nu_{1}(x)=-\frac{1}{p}\right] .
$$

Consider the polynomial $\phi_{\omega}=x^{p}-x-y^{-1} \in K[x]$. Then

$$
\nu_{1}\left(\phi_{\omega}\right)=\min \left\{\nu_{1}\left(x^{p}\right), \nu_{1}(x), \nu_{1}\left(y^{-1}\right)\right\}=\min \left\{-1,-\frac{1}{p},-1\right\}=-1 .
$$

One can show that $\phi_{2}:=x-y^{-1 / p}$ is a (MacLane-Vaquié's) key polynomial for $\nu_{1}$ and we consider the augmented valuation

$$
\nu_{2}:=\left[\nu_{1} ; \nu_{2}\left(\phi_{2}\right)=-\frac{1}{p^{2}}\right] .
$$

Writing $x=\phi_{2}+y^{-1 / p}$, we have

$$
\begin{aligned}
\phi_{\omega} & =\left(\phi_{2}+y^{-1 / p}\right)^{p}-\left(\phi_{2}+y^{-1 / p}\right)-y^{-1} \\
& =\phi_{2}^{p}+y^{-1}-\phi_{2}-y^{-1 / p}-y^{-1}=\phi_{2}^{p}-\phi_{2}-y^{-1 / p} .
\end{aligned}
$$

Hence,

$$
\nu_{2}\left(\phi_{\omega}\right)=\min \left\{\nu_{2}\left(\phi_{2}^{p}\right), \nu_{2}\left(\phi_{2}\right), \nu_{2}\left(y^{-1 / p}\right)\right\}=\min \left\{-\frac{1}{p},-\frac{1}{p^{2}},-\frac{1}{p}\right\}=-\frac{1}{p} .
$$

Continuing the process, one can show that $\phi_{3}:=\phi_{2}-y^{-1 / p^{2}}=x-y^{-1 / p}-y^{-1 / p^{2}}$ is a (MacLane-Vaquié's) key polynomial for $\nu_{2}$ and define

$$
\nu_{3}:=\left[\nu_{2} ; \nu_{3}\left(\phi_{3}\right)=-\frac{1}{p^{3}}\right] \text {. }
$$

Putting $\phi_{2}=\phi_{3}+y^{-1 / p^{2}}$ gives us

$$
\phi_{\omega}=\phi_{3}^{p}-\phi_{3}-y^{-1 / p^{2}} \text { and } \nu_{3}\left(\phi_{\omega}\right)=-\frac{1}{p^{2}} .
$$

We proceed in this manner, until we obtain a sequence of valuations $\left\{\nu_{n}\right\}_{n \in \mathbb{N}}$ for which

$$
\phi_{n+1}=x-\sum_{i=0}^{n} y^{-1 / p^{i}} \in K[x]
$$

is a (MacLane-Vaquié's) key polynomial for $\nu_{n}$ and

$$
\nu_{n+1}:=\left[\nu_{n} ; \nu_{n+1}\left(\phi_{n+1}\right)=-\frac{1}{p^{n+1}}\right] .
$$


Analogously to what we did before, we have

$$
\phi_{\omega}=\phi_{n+1}^{p}-\phi_{n+1}-y^{-1 / p^{n}} \text { and } \nu_{n+1}\left(\phi_{\omega}\right)=-\frac{1}{p^{n}} .
$$

Setting $a_{n}=\sum_{i=0}^{n} y^{-1 / p^{i}}$ we see that $\left\{a_{n}\right\}_{n \in \mathbb{N}} \subseteq K$ is a pseudo-convergent sequence for $\nu_{0}$. Indeed, for $m<n<l$ we have

$$
\nu_{0}\left(a_{n}-a_{m}\right)=\nu_{0}\left(\sum_{i=m+1}^{n} y^{-1 / p^{i}}\right)=-\frac{1}{p^{m+1}}<-\frac{1}{p^{n+1}}=\nu_{0}\left(a_{l}-a_{n}\right) .
$$

Let $\bar{K}$ be the algebraic closure of $K$ inside $k\left(\left(y^{\mathbb{Q}}\right)\right)$ and denote the valuation induced on $\bar{K}$ by the $y$-adic valuation again by $\nu_{0}$. Then $\eta:=\sum_{i=1}^{\infty} y^{-1 / p^{i}} \in \bar{K}$, because it is a root of $\phi_{\omega}$. It is immediate from the definition that $\eta$ is a limit for $\left\{a_{n}\right\}_{n \in \mathbb{N}}$. Hence, any other limit of $\left\{a_{n}\right\}_{n \in \mathbb{N}}$ should be of the form $\eta+\eta^{\prime}$ with $\nu_{0}\left(\eta^{\prime}\right) \geq 0$ and hence cannot belong to $K$. Now, since $\phi_{n+1}=x-a_{n}$, we have $\phi_{n+1}\left(a_{n}\right)=0$ and consequently by (5), we have

$$
\nu_{0}\left(\phi_{\omega}\left(a_{n}\right)\right)=\nu_{0}\left(\phi_{n+1}^{p}\left(a_{n}\right)-\phi_{n+1}\left(a_{n}\right)-y^{-1 / p^{n}}\right)=\nu_{0}\left(-y^{-1 / p^{n}}\right)=-\frac{1}{p^{n}} .
$$

Therefore the value of $\phi_{\omega}$ is not fixed by $\left\{a_{n}\right\}_{n \in \mathbb{N}}$.

We claim that $\phi_{\omega}$ is a polynomial of smallest degree whose value is not fixed by $\left\{a_{n}\right\}_{n \in \mathbb{N}}$. Indeed, it follows from [3] that such a degree must be a power of $p$. Since $\left\{a_{n}\right\}_{n \in \mathbb{N}}$ does not have a limit in $K$, such a degree must be greater than or equal to $p$. Since $\operatorname{deg}\left(\phi_{\omega}\right)=p$, we conclude that $\phi_{\omega}$ has the smallest degree possible.

One can show that the sequence $\left\{\nu_{n}\right\}_{n \in \mathbb{N}}$ is a sequence of augmented valuations on $K[x]$ and that $\phi_{\omega}$ is a limit key polynomial (in Vaquié's language) for $\left\{\nu_{n}\right\}_{n \in \mathbb{N}}$.

6.2. Construction of the second limit key polynomial. Now take $\gamma \in \mathbb{Q} \cup\{\infty\}$ with $\gamma \geq 0$. Since $0>-\frac{1}{p^{n}}=\nu_{n}\left(\phi_{\omega}\right)$ for every $n \in \mathbb{N}$, we can consider the valuation

$$
\nu_{\omega}:=\left[\left\{\nu_{n}\right\}_{n \in \mathbb{N}} ; \nu_{\omega}\left(\phi_{\omega}\right)=\gamma\right] \text {. }
$$

REMARK 6.1. If $\gamma=\infty$, then $\nu_{\omega}$ induces a valuation on $K(\eta)=K[x] /\left(\phi_{\omega}\right)$ which is exactly the valuation given in Theorem 4.3. In this case, the pseudo-convergent sequence $\left\{a_{n}\right\}_{n \in \mathbb{N}}$ can be thought of as a "pseudo-convergent sequence of algebraic type with an algebraic limit" (because in this case $\eta$ is a limit for it).

So far, we have constructed an example where the sequence of key polynomials of order type $\omega$ "is not enough to construct the valuation". In terms of pseudo-convergent sequences, this means that the pseudo-convergent sequence is of algebraic type. We will now continue the construction, starting from the valuation $\nu_{\omega}$ defined by the limit key polynomial $\phi_{\omega}$.

Set $\gamma=0$ and let $\phi_{2 \omega}:=\phi_{\omega}^{p}-y \phi_{\omega}-1$. Then

$$
\nu_{\omega}\left(\phi_{2 \omega}\right)=\min \left\{\nu_{\omega}\left(\phi_{\omega}^{p}\right), \nu_{\omega}\left(y \phi_{\omega}\right), \nu_{\omega}(1)\right\}=\min \{0,1,0\}=0 .
$$

One can show that $\phi_{\omega+1}=\phi_{\omega}-1$ is a (MacLane-Vaquié's) key polynomial for $\nu_{\omega}$ and we can define the valuation

$$
\nu_{\omega+1}:=\left[\nu_{\omega} ; \nu_{\omega+1}\left(\phi_{\omega+1}\right)=\frac{1}{p}\right]
$$


Since $\phi_{\omega}=\phi_{\omega+1}+1$, we have

$$
\begin{aligned}
\phi_{2 \omega} & =\left(\phi_{\omega+1}+1\right)^{p}-y\left(\phi_{\omega+1}+1\right)-1 \\
& =\phi_{\omega+1}^{p}+1-y \phi_{\omega+1}-y-1=\phi_{\omega+1}^{p}-y \phi_{\omega+1}-y .
\end{aligned}
$$

Hence,

$$
\nu_{\omega+1}\left(\phi_{2 \omega}\right)=\min \left\{\nu_{\omega+1}\left(\phi_{\omega+1}^{p}\right), \nu_{\omega+1}\left(y \phi_{\omega+1}\right), \nu_{0}(y)\right\}=1 .
$$

One can prove that

$$
\phi_{\omega+2}:=\phi_{\omega+1}-y^{1 / p}=\phi_{\omega}-1-y^{1 / p}
$$

is a (MacLane-Vaquié's) key polynomial for $\nu_{\omega+1}$. We set

$$
\nu_{\omega+2}:=\left[\nu_{\omega+1} ; \nu_{\omega+2}\left(\phi_{\omega+2}\right)=\frac{1+p}{p^{2}}\right] .
$$

Then

$$
\phi_{2 \omega}=\left(\phi_{\omega+2}+y^{1 / p}\right)^{p}-y\left(\phi_{\omega+2}+y^{1 / p}\right)-y=\phi_{\omega+2}^{p}-y \phi_{\omega+2}-y^{(1+p) / p}
$$

and consequently,

$$
\nu_{\omega+2}\left(\phi_{2 \omega}\right)=\min \left\{\nu_{\omega+2}\left(\phi_{\omega+2}^{p}\right), \nu_{\omega+2}\left(y \phi_{\omega+2}\right), \nu_{0}\left(y^{(1+p) / p}\right)\right\}=\frac{1+p}{p} .
$$

We can construct a sequence of valuations $\left\{\nu_{\omega+n}\right\}_{n \in \mathbb{N}}$ such that

$$
\phi_{\omega+n+1}=\phi_{\omega+n}-y^{\left(1+\ldots+p^{n-1}\right) / p^{n}}=\phi_{\omega}-1-\sum_{i=1}^{n} y^{\left(1+\ldots+p^{i-1}\right) / p^{i}} \in K[x]
$$

is a (MacLane-Vaquié's) key polynomial for $\nu_{\omega+n}$ and

$$
\nu_{\omega+n+1}:=\left[\nu_{\omega+n} ; \nu_{\omega+n+1}\left(\phi_{\omega+n+1}\right)=\frac{1+\ldots+p^{n}}{p^{n+1}}\right] .
$$

Also,

$$
\phi_{2 \omega}=\phi_{\omega+n+1}^{p}-y \phi_{\omega+n+1}-y^{\left(1+\ldots+p^{n}\right) / p^{n}} \quad \text { and } \quad \nu_{\omega+n+1}\left(\phi_{2 \omega}\right)=\frac{1+\ldots+p^{n}}{p^{n}} .
$$

REMARK 6.2. The sequence $\left\{\nu_{\omega+n}\right\}_{n \in \mathbb{N}}$ is an augmented sequence of valuations (in the Vaquié's language) and

$$
\nu_{\omega+n}\left(\phi_{2 \omega}\right)>\nu_{\omega+m}\left(\phi_{2 \omega}\right) \text { if } n>m .
$$

If $\nu$ is a valuation of $K[x]$ such that

$$
\nu\left(\phi_{i}\right)=\nu_{i}\left(\phi_{i}\right) \text { for every } i, 0 \leq i<2 \omega,
$$

then one can show that $\phi_{2 \omega}$ is a (MacLane-Vaquié's) limit key polynomial for $\nu$. Moreover, for each $i, 0 \leq i<2 \omega$, the polynomial $\phi_{i}$ is a key polynomial for $\nu$ and $\nu_{\phi_{i}}=\nu_{i}$.

6.3. Alternative construction. In this section we will present an alternative way of constructing valuations $\nu_{\eta^{\prime}}$ on $K[x]$ from an element $\eta^{\prime} \in k\left(\left(y^{\mathbb{Q}}\right)\right)$. Moreover, we will present an element $\eta^{\prime} \in k\left(\left(y^{\mathbb{Q}}\right)\right)$ such that the valuations $\nu_{i}$ in the previous sections can be obtained by the truncations of $\nu_{\eta^{\prime}}$ on the polynomials $\phi_{i}$. 
Fix $\eta^{\prime} \in k\left(\left(y^{\mathbb{Q}}\right)\right) \backslash K$. Since $k\left(\left(y^{\mathbb{Q}}\right)\right)$ is algebraically closed, we can define a valuation on $K[x]$ by setting

$$
\nu_{\eta^{\prime}}\left(a_{n} x^{n}+\ldots+a_{0}\right):=\nu_{0}\left(a_{n}\right)+\sum_{i=1}^{r} \nu_{y}\left(\eta^{\prime}-\eta_{i}\right) \text { where } a_{n} x^{n}+\ldots+a_{0}=a_{n} \prod_{i=1}^{r}\left(x-\eta_{i}\right) .
$$

In other words, $\nu_{\eta^{\prime}}(p(x))=\nu_{y}\left(p\left(\eta^{\prime}\right)\right)$ for each $p(x) \in K[x]$.

REMARK 6.3. Observe that if $\eta^{\prime}$ is transcendental, then the valuation defined above extends to a valuation on $K(x)$, because in this case, $\operatorname{supp}(\nu)=0$. On the other hand, if $\eta^{\prime}$ is algebraic, then for the minimal polynomial $p_{\eta^{\prime}}$ of $\eta^{\prime}$ over $K$ we have $\nu_{\eta^{\prime}}\left(p_{\eta^{\prime}}\right)=\infty$. Since $p_{\eta^{\prime}}$ is irreducible, we have $\operatorname{supp}(\nu)=\left(p_{\eta^{\prime}}\right)$. Hence, the valuation defined above induces a valuation on $K\left(\eta^{\prime}\right)=K[x] /\left(p_{\eta^{\prime}}\right)$ (see Remark 2.3).

One can prove that if

$$
\eta^{\prime}=\eta+\eta^{\prime \prime} \text { with } \operatorname{supp}\left(\eta^{\prime \prime}\right) \geq 0,
$$

then the truncation of $\nu_{\eta^{\prime}}$ on the polynomial $\phi_{i}, 1 \leq i<\omega$, is exactly $\nu_{i}$. Moreover, if $\eta^{\prime \prime}$ is transcendental over $K$, then $\left\{a_{n}\right\}_{n \in \mathbb{N}}$ is a "pseudo-convergent sequence of algebraic type with a transcendental pseudo-limit" (because $\eta^{\prime}$ is a limit of it).

In order to obtain $\eta^{\prime \prime}$ in expression $(9)$ such that for each $i, \omega \leq i<2 \omega$, the truncation of $\nu_{\eta^{\prime}}$ on $\phi_{i}$ is $\nu_{i}$, we need the following remark.

REMARK 6.4. If

$$
p(x)=x^{p}-x-a_{0}-a_{1}-\ldots-a_{n} \in K[x], a_{0}, \ldots, a_{n} \in K
$$

is an Artin-Schreier polynomial, then all the roots of $p$ are

$$
\eta_{0}+\ldots+\eta_{n}+j, 0 \leq j \leq p-1, \text { where } \eta_{i} \text { is a root of } x^{p}-x-a_{i} .
$$

Let $\eta_{1} \in k$ be a root of $x^{p}-x-1$. Then $\eta+\eta_{1}+j, 0 \leq j \leq p-1$, are all the roots of $\phi_{\omega+1}$. On the other hand, for each $\alpha \in \mathbb{Q}_{>0}$ we see that

$$
\theta_{\alpha}:=-\sum_{i=0}^{\infty} y^{p^{i} \alpha} \in k\left(\left(y^{\mathbb{Q}}\right)\right) \text { is a root of } X^{p}-X-y^{\alpha} .
$$

Hence, if for $n>1$ we set $\eta_{n}:=\theta_{\left(1+\ldots+p^{n-2}\right) / p^{n-1}}$, then

$$
\eta+\eta_{1}+\ldots+\eta_{n}+j, 0 \leq j \leq p-1 \text {, are all the roots of } \phi_{\omega+n} .
$$

Take

$$
\eta^{\prime}=\eta+\eta_{1}-y^{1 / p}-\ldots-y^{\left(1+\ldots+p^{n-1}\right) / p^{n}}-\ldots=\eta+\eta_{1}-\sum_{i=1}^{\infty} y^{\left(1+\ldots+p^{i-1}\right) / p^{i}} .
$$

Then

$$
\nu_{\eta^{\prime}}\left(x-\eta+\eta_{1}+\ldots+\eta_{n}\right)=\nu_{y}\left(\eta^{\prime}-\eta+\eta_{1}+\ldots+\eta_{n}\right)=\frac{1+\ldots+p^{n}}{p^{n+1}}
$$

and

$$
\nu_{\eta^{\prime}}\left(x-\eta+\eta_{1}+\ldots+\eta_{n}+j\right)=0 \text { for every } j, 1 \leq j \leq p-1
$$

Hence

$$
\nu_{\eta^{\prime}}\left(\phi_{\omega+n}\right)=\frac{1+\ldots+p^{n-1}}{p^{n}}=\nu_{\omega+n}\left(\phi_{\omega+n}\right) .
$$

One can show that, in this case, for each $i, 1 \leq i<2 \omega$, the truncation of $\nu_{\eta^{\prime}}$ on $\phi_{i}$ is $\nu_{i}$. 
6.4. Algorithm. The process to construct key polynomials used here is based in an algorithm which is a matter of research at the moment. Our hope is that this algorithm will allow us to give a description of the key polynomials associated to a valuation in a constructive way. Such a description is very important in the program of Spivakovsky to solve the local uniformization problem in positive characteristic.

This algorithm can be described vaguely as follows. Once you have a limit key polynomial $\phi$, there are polynomials which are candidates to be the next limit key polynomial (they can be described in terms of p-polynomials and suitable Newton polygons). Once you fix a candidate $\psi$, one can use an algorithm similar to the Newton's method to approximate roots of a polynomial to obtain the key polynomials $q$, such that $\phi<q<\psi$ (where the order is given by $\phi<q$ if and only if $\epsilon(\phi)<\epsilon(q)$ ). Observe that in the constructions of Section 6.1 we found the polynomials $\phi_{i}, 1 \leq i<\omega$, depending on the polynomial $\phi_{\omega}=x^{p}-x-y^{-1}$. In Section 6.2 , we also constructed the key polynomials $\phi_{\omega+n}$ depending on the polynomial $\phi_{2 \omega}=\phi_{\omega}^{p}-y \phi_{\omega}-1$.

\section{References}

[1] V. Alexandru, N. Popescu, A. Zaharescu, A theorem of characterization of residual transcendental extensions of a valuation, J. Math. Kyoto Univ. 28 (1988), 579-592.

[2] J. Decaup, W. Mahboub, M. Spivakovsky, Abstract key polynomials and comparison theorems with the key polynomials of MacLane-Vaquié, Illinois J. Math. 62 (2018), 253-270.

[3] I. Kaplansky, Maximal fields with valuations I, Duke Math. J. 9 (1942), 303-321.

[4] F.-V. Kuhlmann, Value groups, residue fields and bad places of rational function fields, Trans. Amer. Math. Soc. 356 (2004), 4559-4600.

[5] S. MacLane, A construction for absolute values in polynomial rings, Trans. Amer. Math. Soc. 40 (1936), 363-395.

[6] J. Novacoski, Key polynomials and minimal pairs, J. Algebra 523 (2019), 1-14.

[7] J. Novacoski, M. Spivakovsky, Key polynomials and pseudo-convergent sequences, J. Algebra 495 (2018), 199-219.

[8] M. Spivakovsky, Valuations in function fields of surfaces, Amer. J. Math. 112 (1990), 107156.

[9] M. Vaquié, Extension d'une valuation, Trans. Amer. Math. Soc. 359 (2007), 3439-3481. 